\title{
Elements of Continuum Mechanics and Conservation Laws
}




\section{Elements of}

\section{Continuum Mechanics}

\section{and Conservation Laws}

Sergei K. Godunov and Evgenii I. Romenskii S. L. Sobolev Institute of Mathematics SB RAS Novosibirsk, Russia

Springer Science+Business Media, LLC 
С. К. Годунов, Е. И. Роменский

ЭЛЕМЕНТЫ МЕХАНИКИ СПЛОШНЫХ СРЕД

И ЗАКОНЫ СОХРАНЕНИЯ

Научняя книга (ИДМИ), Новосибирск, 1998

Translated from the Russian with new material added by the authors by Tamara Rozhkovskaya, Novosibirsk, Russia

ISBN 978-1-4419-3399-7 ISBN 978-1-4757-5117-8 (eBook)

DOI 10.1007/978-1-4757-5117-8

(C)2003 Springer Science+Business Media New York

Originally published by Kluwer Academic/Plenum Publishers, New York in 2003

Softcover reprint of the hardcover 1st edition 2003

http://www.wkap.nl/

$\begin{array}{llllllllll}10 & 9 & 8 & 7 & 6 & 5 & 4 & 3 & 2 & 1\end{array}$

A C.I.P. record for this book is available from the Library of Congress

All rights reserved

No part of this book may be reproduced, stored in a retrieval system, or transmitted in any form or by any means, electronic, mechanical, photocopying, microfilming, recording, or otherwise, without written permission from the Publisher, with the exception of any material supplied specifically for the purpose of being entered and executed on a computer system, for exclusive use by the purchaser of the work.

Permissions for books published in Europe: permissions@wkap.nl

Permissions for books published in the United States of America: permissions@wkap.com 


\section{Preface}

This English edition draws on the original work of S. Godunov [5]. This book was published in Russia in 1978 and received the M. A. Lavrent'ev prize of the Russian Academy of Science in 1993. Later, the material of [5] was included in the book by both authors [7, Chapters I-IV] published in 1998. This edition also presents a new concept developed by S. Godunov on the basis of examples due to E. Romenskii. This concept was essentially developed while preparing the English translation of [7] and was intensively discussed with specialists in France, Russia, and USA last year. Four years ago we could only conjecture as to the existence of relationships between the structure of thermodynamical conservation laws and representations of the rotation group. Now, we can say more owing to the very recent results of S. Godunov and V. Gordienko (cf. Appendix and references there).

Chapters I-IV were based on lectures given by S. Godunov at the Physical Department of the Novosibirsk State University. This course was based on the original results of both authors and concerned formulations of differential equations describing high-rate deformations of metals. To study such processes, equations of elasticity should be stated in one zone, whereas equations of hydrodynamics should be used in other zones where, for example, cumulative jets appear. Plastic deformations can occur in transition zones, which causes the residual stresses. The suggested model differs from the equations of elasticity by relaxation terms simulating plastic deformations and transistion of an 
elastic medium to a fluid. This model was often used for numerical purposes in simulating high-rate deformations of metals $[2,6]$.

Using thermodynamics laws and the so-called generalized thermodynamical potentials, we can write differential equations describing physical processes in a special form. The simplest variant of such a model suggested by S. Godunov in 1961 and discussed in Sections 19-22 is well known and widely used at present $[1,3,4,8-10]$.

It should be noted that approaches to systematization of equations simulating processes in continuous media are extensively discussed in the literature. This topic is attractive for many researchers. However, the study is still far from a complete theory and there exist many different and sometimes opposite points of view, which was observed even between the authors here.

Naturally, the concept presented in the Appendix cannot be regarded as a final scheme of formalization of differential equations describing processes in continuous media but the ideas and methods expressed there could be helpful for the further study of these problems and hopefully attract the attention of not only specialists in mechanics and physics but also mathematicians studying solutions to partial differential equations and their properties.

In my opinion, the further development of the theory of thermodynamically compatible conservation laws will lead to very interesting results of general applicability. In addition, an appropriate systematization of various models of physical processes could be also useful in numerical methods

Sergei Godunov

November, 2002

Novosibirsk, Academtown 


\section{Contents}

Chapter I. Elementary Properties of Deformations and Stresses ............

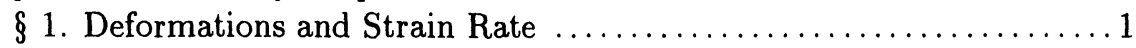

$\S 2$. General Properties of Stresses $\ldots \ldots \ldots \ldots \ldots \ldots \ldots \ldots \ldots \ldots \ldots \ldots$

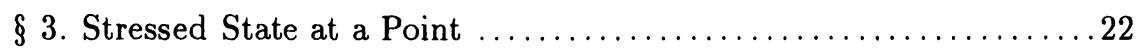

$\S 4$. Isotropic Elastic Medium, Equation of State, and the Murnaghan Formulas ............................29

$\S 5$. Viscous Stresses in an Isotropic Medium .................. 42

$\S 6$. Strain Tensors and Distortion Tensor .....................52

$\S 7$. Murnaghan Formulas in the Tensor Form and Small Deformations ................................... 57

$\S 8$. Moving Medium. Murnaghan Formulas $\ldots \ldots \ldots \ldots \ldots \ldots \ldots \ldots 63$

Chapter II. Effective Elastic Deformation $\ldots \ldots \ldots \ldots \ldots \ldots \ldots \ldots \ldots \ldots \ldots$

$\S 9$. Relaxation of Stresses ...............................71

$\S 10$. Equations for the Metric Effective Elastic Deformation

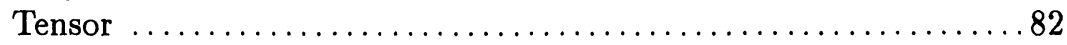

$\S 11$. Compatibility Conditions $\ldots \ldots \ldots \ldots \ldots \ldots \ldots \ldots \ldots \ldots . \ldots 8$

$\S 12$. Description of Relaxation of Shear Stresses by Equations for Effective Distortion .................... 103

$\S 13$. Defects of Crystal Lattice. Review of Notions (Dislocations) ....112

Chapter III. Differential Equations of Dynamical Processes ............ 121

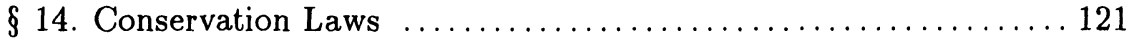

$\S 15$. Consequences of Conservation Laws $\ldots \ldots \ldots \ldots \ldots \ldots \ldots \ldots \ldots$

$\S 16$. Deformation of a Layer and a Bar .................... 130

$\S 17$. Equations for One-Dimensional Nonstationary Processes and Structure of Stationary Waves in a Maxwell Medium ........139 
Chapter IV. Well-Posedness of Differential Equations and

Thermodynamics

$\S 18$. Well-Posedness Conditions for Equations of Elasticity

$\S 19$. Equations of Linear Elasticity as Symmetric Hyperbolic Equations

$\S 20$. Symmetrization of Equations of Gas Dynamics

$\S 21$. Influence of Small Viscosity on the Behavior of Solutions 176

$\S 22$. Well-Posedness, Stability, and Postulates of Phenomenological Thermodynamics

Chapter V. Multi-Dimensional Thermodynamically Compatible

Conservation Laws 203

$\S 23$. Gas Dynamics Equations and Magnetic Hydrodynamics Equations ..................................... 203

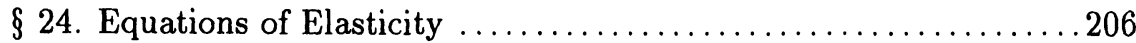

$\S 25$. Structure of Multi-Dimensional Equations .................211

$\S 26$. Symmetric Hyperbolicity in the Three-Dimensional Case . . . . . 215

Appendix. Structure of Thermodynamically Compatible

Systems - S. K. Godunov ............................... 217

$\S 1$. Mathematical Aspects ............................217

$\S 2$. The Simplest Galilei-Invariant Thermodynamically Compatible Systems . . . . . . . . . . . . . . . . . . . . . . . . . . . . . . 219

$\S 3$. Methods of Constructing Equations .................... 227

$\S 4$. Some Facts of the Theory of Representations of Orthogonal Transformations of Three-Dimensional Space .............238

$\S 5$. The Clebsch-Gordan Coefficients .................... 245

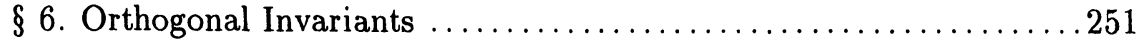

Literature .......................................... 255

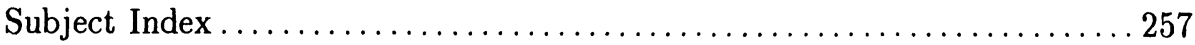

